\title{
HCC occurrence after DAA treatments: molecular tools to assess the post-treatment risk and surveillance
}

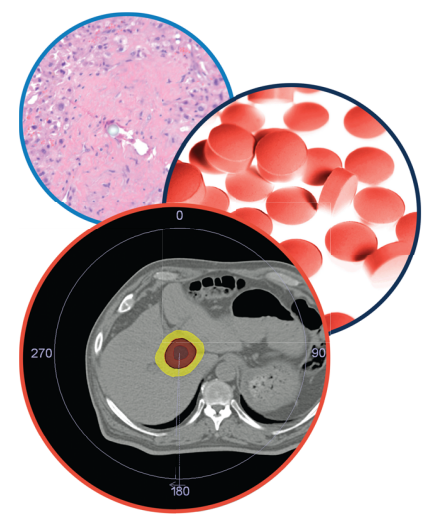

Hepatic Oncology

\author{
Devis Pascut*,1(iD), Muhammad Yogi Pratama ${ }^{1,2}$ \& Claudio Tiribelli1 (iD) \\ ${ }^{1}$ Fondazione Italiana Fegato - ONLUS, Liver Research Center, AREA Science Park, ss14, Km163.5, Basovizza, 34149 Trieste, Italy \\ ${ }^{2}$ Universitas Hasanuddin, Faculty of Medicine, Makassar, 90245, Indonesia \\ *Author for correspondence: devis.pascut@fegato.it
}

\begin{abstract}
"despite any controversy regarding whether DAA increase the risk of de novo HCC occurrence after achieving SVR, there is an urgent need for a risk stratification strategy that combines baseline characteristics of patients, the pre-existing HCC risk factors and serum biomarkers before and after DAA treatment protocols"
\end{abstract}

First draft submitted: 31 March 2020; Accepted for publication: 6 May 2020; Published online: 22 June 2020

Keywords: biomarkers • DAA • direct-acting antiviral • HCC • HCV

The perspective of hepatitis $\mathrm{C}$ virus (HCV) therapy has dramatically changed over the years after the introduction of direct-acting antiviral (DAA) therapy, which increased the sustainable viral response rate (SVR) up to $90 \%$ with better tolerance and effectiveness in clinical practice as compared with interferon-based regimens [1]. However, despite the excellent efficacy and extensive studies, alarming reports from two retrospective studies, conducted in 2016 in Spain and Italy [2,3], suggested an increased risk of hepatocellular carcinoma (HCC) occurrence and recurrence after DAA treatment in patients, triggering the debate on the safety profile of DAA and its correlation to HCC development. Nonetheless, some criticisms regarding the absence of control groups, sample size or short-follow-up periods have been raised in some studies.

A meta-analysis conducted by Waziry et al. in 2017 estimated no significant HCC occurrence cases in both DAA-treated or interferon-treated patients following SVR [4]. Indeed, several reports underlined the efficacy of DAA in significantly reducing the risk of HCC in patients with SVR as compared with those with either treatment failure or no treatment $[1,5,6]$. However, what emerges from those studies is that the DAA-induced SVR reduces the risk of HCC occurrence, without eliminating it. This includes patients with other risk factors, such as age, gender and cirrhosis. In particular, patients with cirrhosis with long exposure to the virus, can still be considered at risk, even after the achievement of SVR. Hamdane $e t$ al. described how epigenetic alterations induced by chronic HCV infection persist even after viral clearance and were further associated with HCC risk [7]. Liver alteration was also evident in early studies conducted by Kono et al., observing sustained abnormal ALT and AFP levels, especially in F3-F4 patients (F3: severe fibrosis, characterized by fibrotic bridging across lobules, between portal areas and between portal areas and central veins; F4: cirrhosis), even after the achievement of SVR. Multivariate analysis identified pre-treatment low albumin levels and fibrosis 4 (FIB-4) index as independent predictive factors for the sustained AFP after SVR. At the same time, the fatty liver presence was associated with both sustained abnormal AFP and ALT levels after SVR [8] suggesting that the persistence of hepatocyte damage and regeneration mechanisms might lead to HCC development. The oxidative stress present in the fatty liver might also be responsible for DNA damage that foster carcinogenesis [8]. Confirming the results of Kono et al., Watanabe et al. identified FIB-4 index $\geq 4.0$ and albumin $\leq 3.8 \mathrm{~g} / \mathrm{dl}$ at the beginning of DAA treatment and a FIB- 4 index $\geq 4.0$ and AFP $\geq 6.0$ at the end of DAA treatment as independent predictors for HCC occurrence [9]. Moreover, despite the correlation between HCC risk with fibrosis index, recent data suggested the presence of liver steatosis in HCV patients as a major predictor of mortality and HCC occurrence in patients who achieved SVR following DAA treatment regardless of fibrosis [10].

Future Medicine 
Other studies suggested alternative serum biomarker candidates associated with HCC occurrence, focusing on the alteration of the immunological profiles during and after DAA-induced HCV elimination [11]. In 2017, Debes et al. identified a set of circulating immune mediators measured before DAA treatment: MIG, IL-22, TRAIL, APRIL, VEGF, IL-3, TWEAK, SCF and IL-21A. These markers were able to identify patients who developed de novo HCC [12]. Subsequent studies reported a significantly higher levels of IL- 4 and IL-13 before and after DAA treatments in patients who developed HCC, suggesting a pro-oncogenic immune profile in those patients at risk.

The alteration of inflammatory and anti-inflammatory balance might affect antitumor surveillance contributing to the development of HCC. For example, the persistence of T-reg and the decrease of natural killer group 2 member D cells, after DAA treatments, might be one of the causes of early HCC occurrence after SVR, leading to the proliferation of undetectable dysplastic nodules already present within the damaged liver [13]. Also, the increase in VEGF and liver Ang2 found in the serum of DAA-treated patients might further sustain the tumor growth by promoting neo-angiogenesis $[14,15]$. These alterations in the immunological status and the consequent changes in cytokines and chemokines profiles before and after DAA treatment might represent a marker which could aid in the identification of patients at risk of HCC development.

Circulating miRNA might represent a promising tool to assess pre DAA-treatment HCC-risk and post DAAtreatment surveillance. In a retrospective study, we evaluated the association of circulating miRNA biomarkers with the risk of HCC occurrence and suggested that patients who develop HCC had a pre-existing risk, even before the DAA therapy initiation [16]. The alterations of circulating miRNAs were associated to HCC risk before therapy initiation and persisted after viral clearance [16], suggesting that the risk of HCC occurrence may be identifiable even before initiation of DAA therapy.

Other studies evaluated the potential use of alternative circulating biomarkers for patients' surveillance. Serum sphingolipids, for instance, were able to predict de novo HCC in HCV cirrhotic patients with SVR with an accuracy greater than AFP [17]. In another study, Yasui et al. showed that the level of $\mathrm{WFA}^{+}-\mathrm{M} 2 \mathrm{BP}$ might be used as postDAA treatment biomarker for assessing the risk of HCC development [18]. WFA ${ }^{+}-\mathrm{M} 2 \mathrm{BP}$ is secreted by hepatic stellate cells and can induce Mac-2 expression in Kupffer cells, resulting in the activation of stellate cells and the production of fibrogenic chemokines, actively contributing in the pro-oncogenic environment [19].

Based on the previously discussed studies, circulating biomarkers hold promise as useful tools to be included in risk-predicting algorithms for better patient stratification. The benefit of being noninvasive approach, easy to measure and cost effective, thus facilitating serial sampling and the monitoring of dynamic changes during patients' follow-up, demonstrate the importance of circulating biomarkers for setting the basis of personalized evidence-based clinical strategies.

Since SVR achievement does not eliminate the risk of HCC, current clinical guidelines still recommend surveillance to be conducted in each individual. However, despite the proven benefits, this strategy might not be feasible in a universal setting. It was estimated that 6 -month programs of surveillance with ultrasound and AFP in cirrhotic cases cost 753,226 USD, a sum totally unfeasible for low-income countries [20]. Therefore, a more specific and cost-efficient surveillance system is needed to stratify patients according to their risk factors, such as advanced fibrosis and cirrhosis, for instance, especially in consideration that the vast majority of HCC occurrence episodes after SVR were observed within 12-24 months [1,3]. Thus, despite any controversy regarding whether DAA increase the risk of de novo HCC occurrence after achieving SVR, there is an urgent need for a risk stratification strategy that combines baseline characteristics of patients, the pre-existing HCC risk factors and serum biomarkers before and after DAA treatment protocols.

However, despite this enormous effort in the biomarker research field, we are conscious that only few are currently utilized in decision-making algorithms. Considering the great complexity of the field, there is a crucial need to develop a shared approach in biomarker discovery and validation studies that accelerates the diffusion of newly discovered biomarkers into clinical practice.

Author contributions

All the authors contributed to text conceptualization, writing and proofreading. 
entity with a financial interest in or financial conflict with the subject matter or materials discussed in the manuscript apart from those disclosed.

No writing assistance was utilized in the production of this manuscript.

\section{Open access}

This work is licensed under the Attribution-NonCommercial-NoDerivatives 4.0 Unported License. To view a copy of this license, visit http://creativecommons.org/licenses/by-nc-nd/4.0/

\section{References}

1. Welzel TM, Nelson DR, Morelli G et al. Effectiveness and safety of sofosbuvir plus ribavirin for the treatment of HCV genotype 2 infection: results of the real-world, clinical practice HCV-TARGET study. Gut 66(10), 1844-1852 (2017).

2. Reig M, Mariño Z, Perelló $\mathrm{C}$ et al. Unexpected high rate of early tumor recurrence in patients with HCV-related HCC undergoing interferon-free therapy. J. Hepatol. 65(4), 719-726 (2016).

3. Conti F, Buonfiglioli F, Scuteri A et al. Early occurrence and recurrence of hepatocellular carcinoma in HCV-related cirrhosis treated with direct-acting antivirals. J. Hepatol. 65(4), 727-733 (2016).

4. Waziry R, Hajarizadeh B, Grebely J et al. Hepatocellular carcinoma risk following direct-acting antiviral HCV therapy: a systematic review, meta-analyses, and meta-regression. J. Hepatol. 67(6), 1204-1212 (2017).

5. Mendizabal M, Piñero F, Ridruejo $\mathrm{E}$ et al. Disease progression in patients with hepatitis $\mathrm{C}$ virus infection treated with direct-acting antiviral agents. Clin. Gastroenterol. Hepatol. doi:10.1016/j.cgh.2020.02.044 (2020) (Epub ahead of print).

6. Ioannou GN, Green PK, Berry K. HCV eradication induced by direct-acting antiviral agents reduces the risk of hepatocellular carcinoma. J. Hepatol. 68(1), 25-32 (2017).

7. Hamdane N, Jühling F, Crouchet $\mathrm{E}$ et al. HCV-induced epigenetic changes associated with liver cancer risk persist after sustained virologic response. Gastroenterology 156(8), 2313-2329.e7 (2019).

8. Kono M, Nishida N, Hagiwara $\mathrm{S}$ et al. Unique characteristics associated with sustained liver damage in chronic hepatitis $\mathrm{C}$ patients treated with direct acting antivirals. Dig. Dis. 35(6), 556-564 (2017).

9. Watanabe $\mathrm{T}$, Tokumoto $\mathrm{Y}$, Joko $\mathrm{K}$ et al. Predictors of hepatocellular carcinoma occurrence after direct-acting antiviral therapy in patients with hepatitis C virus infection. Hepatol. Res. 49(2), 136-146 (2019).

10. Peleg N, Issachar A, Sneh Arbib O et al. Liver steatosis is a major predictor of poor outcomes in chronic hepatitis $\mathrm{C}$ patients with sustained virological response. J. Viral Hepat. 26(11), 1257-1265 (2019).

11. Holmes JA, Carlton-Smith C, Kim AY et al. Dynamic changes in innate immune responses during direct-acting antiviral therapy for HCV infection. J. Viral Hepat. 26(3), 362-372 (2019).

12. Debes JD, van Tilborg M, Groothuismink ZMA et al. Levels of cytokines in serum associate with development of hepatocellular carcinoma in patients with HCV infection treated with direct-acting antivirals. Gastroenterology 154(3), 515-517.e3 (2018).

13. Chu PS, Nakamoto N, Taniki N et al. On-treatment decrease of NKG2D correlates to early emergence of clinically evident hepatocellular carcinoma after interferon-free therapy for chronic hepatitis C. PLoS ONE 12(6), e0179096-e0179096 (2017).

14. Faillaci F, Marzi L, Critelli R et al. Liver angiopoietin-2 is a key predictor of de novo or recurrent hepatocellular cancer after hepatitis $\mathrm{C}$ virus direct-acting antivirals. Hepatology 68(3), 1010-1024 (2018).

15. Villani R, Facciorusso A, Bellanti F et al. DAAs rapidly reduce inflammation but increase serum VEGF level: a rationale for tumor risk during anti-HCV treatment. PLoS ONE 11(12), e0167934 (2016).

16. Pascut D, Cavalletto L, Pratama MY et al. Serum miRNA are promising biomarkers for the detection of early hepatocellular carcinoma after treatment with direct-acting antivirals. Cancers (Basel) 11(11), pii: E1773 (2019).

17. Mücke VT, Thomas D, Mücke MM et al. Serum sphingolipids predict de novo hepatocellular carcinoma in hepatitis $\mathrm{C}$ cirrhotic patients with sustained virologic response. Liver Int. 39(11), 2174-2183 (2019).

18. Yasui Y, Kurosaki M, Komiyama Y et al. Wisteria floribunda agglutinin-positive Mac-2 binding protein predicts early occurrence of hepatocellular carcinoma after sustained virologic response by direct-acting antivirals for hepatitis C virus. Hepatol. Res. 48(13), 1131-1139 (2018).

19. Bekki Y, Yoshizumi T, Shimoda S et al. Hepatic stellate cells secreting WFA+ -M2BP: its role in biological interactions with Kupffer cells. J. Gastroenterol. Hepatol. 32(7), 1387-1393 (2017).

20. Ruggeri M. Hepatocellular carcinoma: cost-effectiveness of screening. A systematic review. Risk Manag. Healthc. Policy 5, 49-54 (2012). 